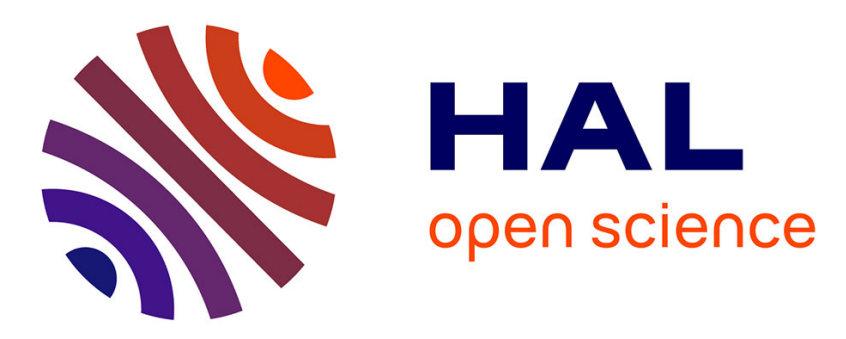

\title{
Review of PSF reconstruction methods and application to post-processing
}

Olivier Beltramo-Martin, Sam Ragland, Romain Fétick, Carlos Correia, Trent Dupuy, Giulian Fiorentino, Thierry Fusco, Laurent Jolissaint, Sebastian

Kamann, Antonino Marasco, et al.

\section{To cite this version:}

Olivier Beltramo-Martin, Sam Ragland, Romain Fétick, Carlos Correia, Trent Dupuy, et al.. Review of PSF reconstruction methods and application to post-processing. SPIE Astronomical Telescopes + Instrumentation 2020, Dec 2020, Online, United States. pp.114480A, 10.1117/12.2560805 . hal03240495

\section{HAL Id: hal-03240495 \\ https://hal.science/hal-03240495}

Submitted on 31 May 2021

HAL is a multi-disciplinary open access archive for the deposit and dissemination of scientific research documents, whether they are published or not. The documents may come from teaching and research institutions in France or abroad, or from public or private research centers.
L'archive ouverte pluridisciplinaire HAL, est destinée au dépôt et à la diffusion de documents scientifiques de niveau recherche, publiés ou non, émanant des établissements d'enseignement et de recherche français ou étrangers, des laboratoires publics ou privés. 


\title{
Review of PSF reconstruction methods and application to post-processing
}

\author{
O. Beltramo-Martin ${ }^{\mathrm{a}}$, S. Ragland ${ }^{\mathrm{b}}$, R. Fétick ${ }^{\mathrm{c}, \mathrm{a}}$, C. Correia ${ }^{\mathrm{d}}$, T. Dupuye,f, G. Fiorentino ${ }^{\mathrm{g}}$, T. $^{\mathrm{e}}$. \\ Fusco $^{\text {c,a }}{ }^{\text {, L. Jolissaint }}{ }^{h}$, S. Kamann ${ }^{\mathrm{i}}$, A. Marasco ${ }^{\mathrm{j}}$, D. Massari ${ }^{\mathrm{k}, \mathrm{l}}$, B. Neichel ${ }^{\mathrm{a}}$, L. Schreiber ${ }^{\mathrm{k}}$, and \\ P. Wizinowich ${ }^{\mathrm{b}}$ \\ ${ }^{a}$ Aix Marseille Univ., CNRS, CNES LAM, 38 rue F. Joliot-Curie, 13388 Marseille, France \\ ${ }^{\mathrm{b}}$ W. M. Keck Observatory, 65-1120 Mamalahoa Hwy, Kamuela, HI 96743 \\ ${ }^{\mathrm{c}}$ ONERA, DOTA, Paris Saclay University, F-92320 Châtillon, France \\ d Space ODT, Rua Adolfo Casais Monteiro, 65, 4050-014 Porto, Portugal \\ eInstitute for Astronomy, University of Edinburgh, Blackford Hill, Edinburgh, EH9 3HJ, United Kingdom \\ ${ }^{\mathrm{f}}$ Gemini Observatory, Northern Operations Center, 670 N. A'ohoku Place, Hilo, HI 96720, USA \\ gINAF-Osservatorio Astronomico di Roma, via Frascati 33, 0040 Monte Porzio Catone, Italy \\ ${ }^{\mathrm{h}}$ University of Applied Sciences Western Switzerland, 1401 Yverdon-les-Bains, Switzerland \\ ${ }^{i}$ Astrophysics Research Institute, Liverpool John Moores University, IC2, Liverpool Science Park, 146 Brownlow Hill, Liverpool \\ L3 5RF, United Kingdom \\ jINAF - Osservatorio Astrofisico di Arcetri, Largo E. Fermi 5, 50127 Firenze, Italy \\ k INAF - Osservatorio di Astrofisica e Scienza dello Spazio di Bologna, Via Gobetti 93/3, I-40129 Bologna, Italy \\ ${ }^{1}$ University of Groningen, Kapteyn Astronomical Institute, NL-9747 AD Groningen, The Netherlands
}

\begin{abstract}
Determining the PSF remains a key challenge for post adaptive-optics (AO) observations regarding the spatial, temporal and spectral variabilities of the AO PSF, as well as itx complex structure. This paper aims to provide a non-exhaustive but classified list of techniques and references that address this issue of PSF determination, with a particular scope on PSF reconstruction, or more generally pupil-plane-based approaches. We have compiled a large amount of references to synthesize the main messages and kept them at a top level. We also present applications of PSF reconstruction/models to post-processing, more especially PSF-fitting and deconvolution for which there is a fast progress in the community.
\end{abstract}

Keywords: Adaptive optics, post-processing

\section{RATIONALE OF THIS REVIEW}

The determination of the point spread function (PSF) is a must in astronomical data processing. Indeed, knowledge of the PSF is critical to estimate the astrophysical signal (photometry/astrometry, extended objects surface topology, or galaxy kinematics, ...) through a post-processing pipeline, that relies on model-fitting or deconvolution for instance. Several issues must be faced to achieve an accurate PSF estimation, in particular field crowding, poor $\mathrm{S} / \mathrm{N}$ or lack of PSF calibrators, that are strengthened in presence of Adaptive-optics (AO) due to spatio-temporal variability of the PSF. Moreover, the emergence of integral field spectrographs calls for considering 3D tools that also model the PSF spectral diversity. In order to overcome existing limits in the AO PSF determination, a constant effort has been deployed for decades in PSF reconstruction (PSF-R) approaches that aim to estimate the PSF from AO control loop data, e.g. AO wavefront measurements and commands. So far, PSF-R lacks of operational uses and science verification owing to dedicated expertise needed and data flow and storage management. A step further must be achieved to enable PSF-R, especially for Giant Segmented Mirrors Telescopes (GSMT). Our first goal is to provide a standardized categorization of the techniques that use focal-plane or pupil-plane data or both jointly combined (hybrid) so as to gain insight into the advantages and shortcomings of such techniques, with regard to post-processing requirements in terms of PSF knowledge. Secondly, I will summarize the ongoing and upcoming actions that are taken by the community for enabling the implementation of PSF-R facilities.

Further author information: (Send correspondence to O.B.M.)

D.M.: E-mail: olivier.beltramo-martin@lam.fr 


\section{EXISTING PSF DETERMINATION TECHNIQUES}

\subsection{Landscape}

In the following, we will use the keyword PSF determination as the ensemble of techniques that aim to providing a 2D representation of the PSF from any contextual data. Among them, we distinguish two top-level families as summarized in Fig. 1 :

- Focal-plane based techniques: all methods that use astronomical images to identify the PSF, that is that the input is a PSF or an extended object convolved by a PSF. Extraction and best-fitting methods are the most common techniques, thanks to their flexibility and the absence of AO expertise needed. ${ }^{1-3}$ They rely usually on a four-part process that consists of (i) correct frames from dark, backgrounds, flat field and distorsions ${ }^{4}$ (ii) detect stars and extract PSFs from the entire field or sub-fields to account for PSF spatial variations (iii) adjust a parametric model over extracted images or stack them to estimate a PSF function (iv) use the PSF model for science post-processing (deconvolution, photometry/astrometry, ...). However, these methods are sensitive to the image noise and source confusion and require the presence of a point-like source within the scientific field ${ }^{5-7}$ calling for consideration of model-fitting instead.

- PSF fitting/interpolation: extracted point sources images and calibrating a PSF model to either deblend sources that overlap or provide a PSF at any position in the field/spectrum.

- PSF marginal estimation and deconvolution: retrieve the PSF from the image of an extended object assuming some priors on the object (and the PSF).

- PSF classification: estimate the PSF from classification methods, such as PCA, supervised classification, and machine-learning/deep-learning -based approaches generally.

- Pupil-plane based techniques : all methods that use wavefront measurements and/or models of wavefront propagation. The input is a time-series of wavefront measurements or integrated parameters derived from wavefront measurements.

- PSF simulation: end-to-end simulations based on integrated atmospheric and system parameters. They allow to include any non-linear effects and rely on wavefront propagation theory. Powerful tools have been developed such as OOMAO, ${ }^{8}$ COMPASS,${ }^{9}$ DASP,${ }^{10}$ PASSATA,,${ }^{11}$ SOAPY, ${ }^{12}$ YAO,${ }^{13}$ OCTOPUS ${ }^{14}$ and many others.

- PSF analytical models: From integrated parameters derived from the AO telemetry, one may use analytical models ${ }^{15-17}$ to infer the PSF as well as the PSF spatial variations due to the anisoplanatism for instance ${ }^{18-20}$

- PSF reconstruction: Estimation of the PSF using AO control loop data or integrated parameters estimated from those. PSFR involves several approximations to derive the PSF from the residual phase power spectrum density.

- Hybrid techniques: all methods that uses jointly astronomical images, wavefront measurements and analytical models

Pupil-plane based techniques can only retrieve the spatial frequencies that are effectively measured by the AO wavefront-sensors (WFS). All non-common path aberrations (NCPA) or field-dependent static aberrations must be calibrated independently, which call for dedicated engineering time. On the contrary, focal-plane based techniques permit to recover a noisy representation of the real PSF including atmospheric and instrumental aberrations, but at specific positions in the field and/or for a particular wavelength. In order to be able to characterize the PSF in a whole field or over broadband observation, model of spatial and spectral PSF variabilities are needed. The PSF determination technique that works best the exploitation of a given astronomical image is obviously science-case dependent. We give in the current section an overview on recent approaches that were developed and successfully validated on-sky, which are the analytical PSF model presented in, ${ }^{21,22}$ the PSF reconstruction ${ }^{23-26}$ and the hybrid PSF reconstruction. ${ }^{27,28}$ 


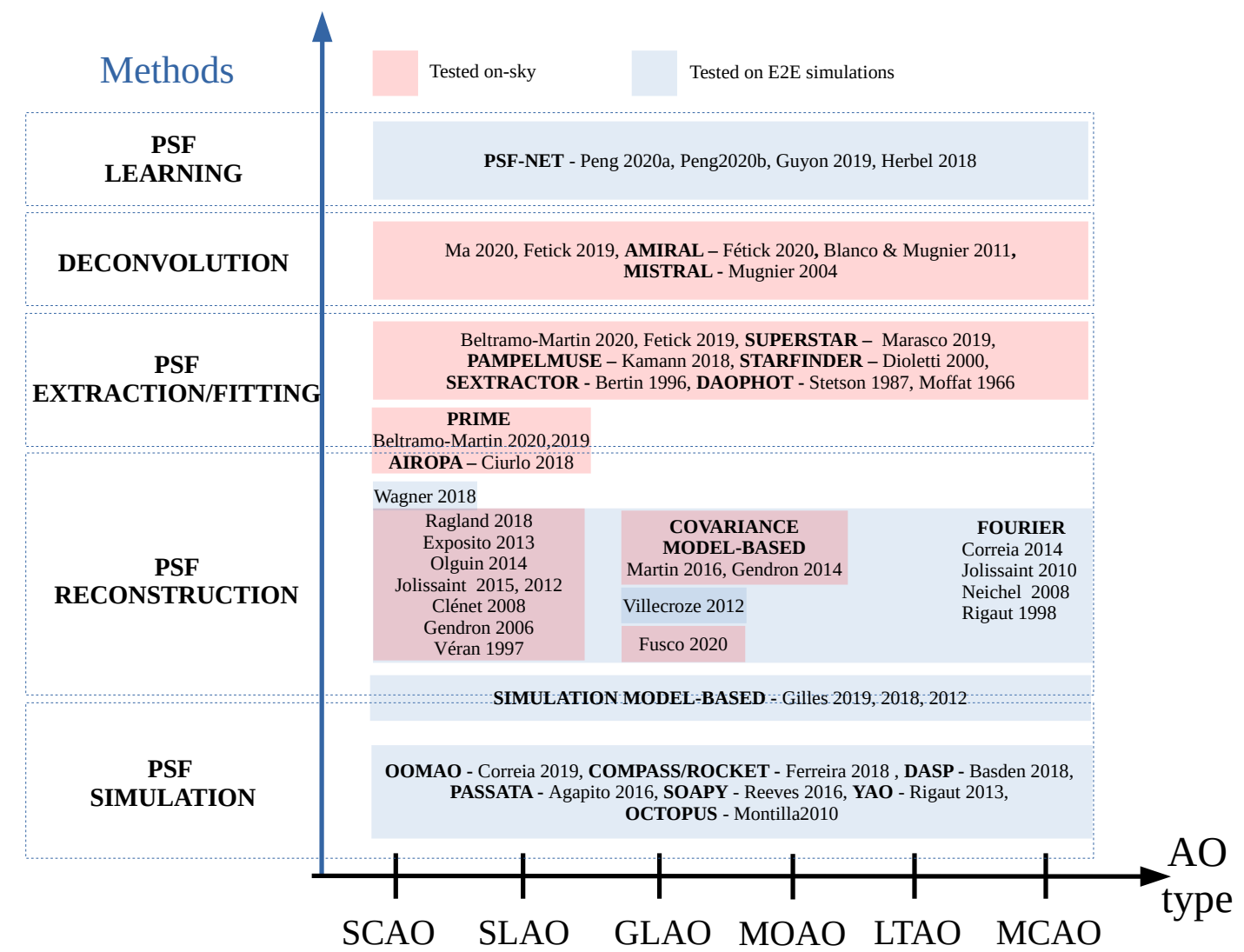

Figure 1: Summary of existing PSF determination techniques and associated references.

\subsection{Scope on advanced PSF models and reconstruction techniques}

In advanced PSF models and PSF reconstruction, the PSF $h(\boldsymbol{\alpha})$ is commonly approximated ${ }^{29}$ as a convolution of an instrumental contribution $\tilde{h}_{t e l}$ with a atmospheric residual term $\tilde{h}_{\mathrm{AO}}$ as follows:

$$
h(\boldsymbol{\alpha})=\mathcal{F}^{-1}\left[\tilde{h}_{t e l}(\boldsymbol{\rho} / \lambda) \tilde{h}_{\mathrm{AO}}(\boldsymbol{\rho} / \lambda)\right]
$$

where $\mathcal{F}^{-1}[x]$ is the inverse 2D Fourier transform of $x$.

The first term $\tilde{h}_{t e l}$ in Eq. 1 is obtained by the autocorrelation of the static phase (the amplitude is assumed to be uniform across the pupil) within the telescope pupil. As a consequence, the pupil-plane based approaches requires the calibration of the pupil masks and the static aberrations (e.g., primary mirror cophasing errors, NCPA, field-dependent aberrations for instance), contrary to focal-plane methods for which all these information are encoded into the data. Then, we already see that achieving pupil-plane-based PSF determination is a challenge and requires an expertise on the telescope, $\mathrm{AO}$ system and the post- $\mathrm{AO}$ instruments.

The calibration of instrumental aberrations, through phase diversity approaches, ${ }^{30-32}$ focal plane sharpening $^{33}$ techniques, or even Deep-learning strategies, ${ }^{34,35}$ is particularly challenging. First of all, sampling the instrumental aberrations across the fov is feasible by the use of internal fibers within the system, ${ }^{36,37}$ but can be potentially time consuming and requires direct control of the system. In other words, this must be anticipated as part of a PSF reconstruction project and can not be easily scheduled at short times scales. Another issue with static aberrations occurs with on-sky calibration as the PSF combines the instrumental part plus the residual atmospheric contribution that blurs the instrumental signature in the data. Current on-sky tests usually relies on the acquisition of long-exposure pairs of in and out-of-focus images, which can be particularly telescope time consuming, ${ }^{25,26}$ especially if one wants to map the instrumental aberrations with respect to the telescope 
elevation. Regardless the pupil-plane-based PSF determination approach, the calibration of static aberrations is mandatory to achieve very accurate (better than a few percents of accuracy on PSF figures of merits) PSF determination. Moving to pupil-plane based techniques is a major change of paradigm: it is far beyond from the sole processing of focal-plane images and involves multi-disciplinary expertise and hands-on work on the system to achieve successful PSF determination.

The second term in Eq. 1 is systematically calculated from ${ }^{29}$

$$
\tilde{h}_{\mathrm{AO}}(\boldsymbol{\rho} / \lambda)=\exp \left(\left(\frac{2 \pi}{\lambda}\right)^{2} \times\left(\mathcal{B}_{\mathrm{AO}}(\boldsymbol{\rho})-\mathcal{B}_{\mathrm{AO}}(\mathbf{0})\right)\right),
$$

where $\mathcal{B}_{\mathrm{AO}}$ is the covariance function of the residual optical path difference downstream AO correction. There are multiple branches that go from this point to the PSF and we draw here an non-exhaustive list.

- Fourier model-based approach. This consists of modeling how spatial frequencies of atmospheric phase disturbances propagate from turbulent layers to the detector focal plane. This requires to describe analytically each component of the AO system so as to understand how a spatial frequency is measured and reconstructed through the wavefront-sensing process. ${ }^{15-17,38,39}$ The covariance function is then obtained through the Wiener-Khintchine theorem that assures that if the residual phase is stationary, we have:

$$
\mathcal{B}_{\mathrm{AO}}(\boldsymbol{\rho})=\mathcal{F}^{-1}\left[\mathcal{W}_{\mathrm{AO}}(\boldsymbol{k})\right]
$$

where $\mathcal{W}_{\mathrm{AO}}$ is the $\mathrm{AO}$ residual phase Power Spectrum Density (PSD). This latter is split into multiple terms that account for different physical limitations, such as the DM cut-off frequency that creates the seeinglimited PSF halo, or the servo-lag error that models how the system latency combined with the atmosphere temporal characteristics create a PSF elongation. The Fourier calculation assumes intrinsically that the residual phase is spatially stationary, meaning its variance remains identical regardless of the position within the pupil plane. which does not apply for modeling the cone effect, although some techniques can be deployed to mitigate this issue. ${ }^{16}$ The Fourier-based approach is very fast to compute (less than $0.5 \mathrm{~s}$ on a standard CPU with 200 pixels to describe a $8 \mathrm{~m}$ pupil) and requires only a few parameters (seeing, $C_{n}^{2}$ profile, DM conjugation altitude, LGS asterisms,...), which is very useful for predicting AO performance with respect to the system design and site observing conditions.

This technique is also a good representation of the post-AO PSF in poor correction regime, as it has been applied on MUSE WFM. ${ }^{23}$ For such systems that address the AO-assisted observations of very large fov (¿1') and rely on a GLAO system, the Fourier-based model has been shown to produce an estimation of PSF figures of merits (FWHM, ensquared energy) at the level of 10-20\% as presented in Fig. 2. At this level of correction (factor 2 improvement of the seeing), the calibration of static aberrations and complex models of the AO system were not necessary to meet the science requirements, and consequently, regarding the this method is an analytical framework, the Fourier approach offers the feasibility to design an operational and robust pipeline from the minimal amount of information from the telemetry.

- Covariance model-based approach. This is a similar approach that involves the same hypothesis on atmosphere statistics. Similarly to the Fourier method, the AO residual is split into error terms (actually the same physical processes) but focuses on modeling the auto- and cross-correlation of those. ${ }^{9,40-42}$ In the case of a point-wise description of the residual phase over $n \times n$ samples, this covariance is calculated over $n^{2} \times n^{2}$ pixels, which means that we derive the covariance of each phase sample with all others. If the phase is spatially stationary, this covariance matrix would have a Toeplitz structure with only $n \times n$ non-redundant elements, and we would not lose information by averaging redundant baselines to get a covariance map, which is connected to the PSD through the Wiener-Khintchine theorem. Compared to the PSD approach, the covariance model-based calculation allows a simpler calculation of the cross-terms, separates properly the tip-tilt modes from the other modes, and accounts correctly for the LGS cone effect, DM spatial filtering and phase spatial non-stationarity in general, which reveal to be particularly relevant in the case of PSF reconstruction for tomographic systems. 

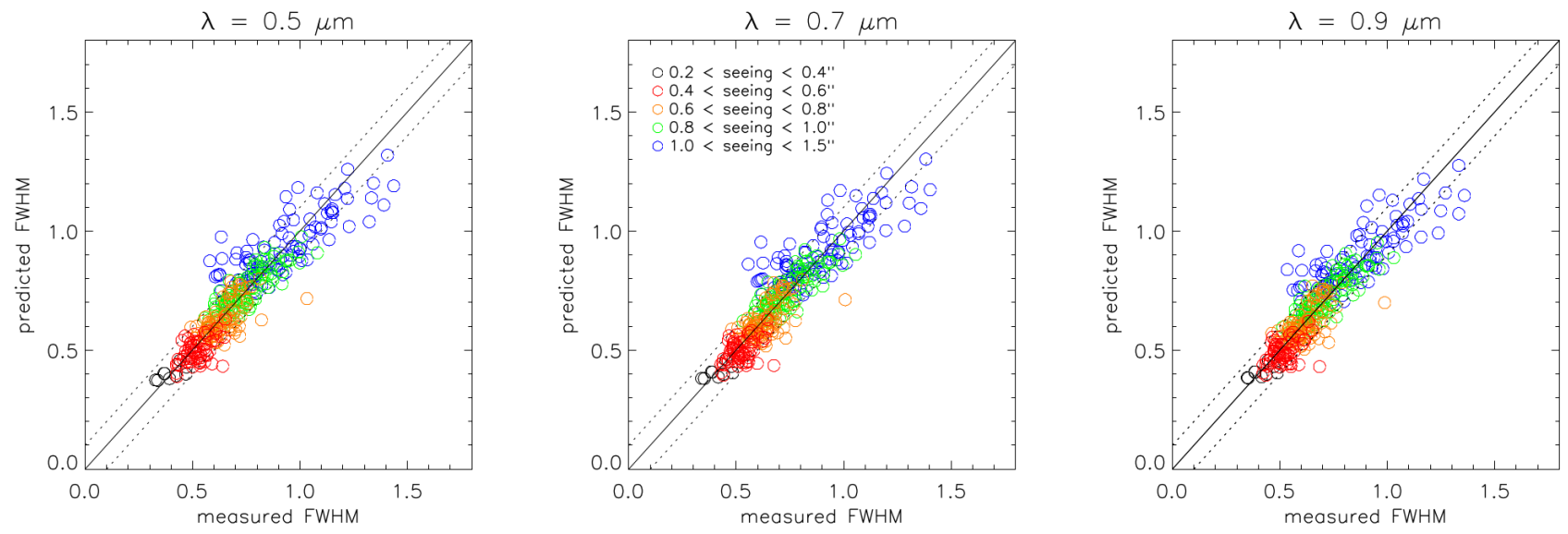

Figure 2: Estimated FWHM from the Fourier reconstruction versus measurements obtained over 94 MUSE WFM PSFs. Three spectrum channels are considered here and color indicates the seeing conditions. ${ }^{23}$

- Reconstruction approach. The techniques presented above degrade the information contained in the telemetry down to few integrated parameters only. With the reconstruction approach, originally introduced by Véran, ${ }^{43} \mathcal{B}_{\mathrm{AO}}$ in Eq. 3 is derived from the empirical covariance of wavefront measurements, which contains more information (second order statistical moments) than the sole integrated parameters. This strategy generally allows improved PSF reconstruction accuracy despite the similar mathematical formalism. On-sky tests have been achieved through the two past decades, especially on PUEO ${ }^{43} \mathrm{NACO},{ }^{44}$ Keck $^{24,26}$ or CANARY, ${ }^{40}$ that showed that reconstructing the PSF at the few percent level is doable. W.M. Keck Observatory has particularly pushed for enabling an accurate PSF reconstruction for the Keck SCAO-assisted observation, with estimation errors pushed down to few percents as presented in Fig. 3. Major limitations seem to be the calibration of static or quasi-static aberrations (NCPA, segment differential piston,...) as well as optical gains variations (quad cell) that must be deeply characterized to break through this limit. There is currently no such PSF reconstruction verification on-sky for LTAO and MCAO systems. This is hypothetically due to lack of access to on-sky AO telemetry data from such operating systems (MUSE NFM, GeMS) and the need of complex recipes to model the PSF across the field. ${ }^{45}$ Note that this strategy is also envisioned for solar AO systems*
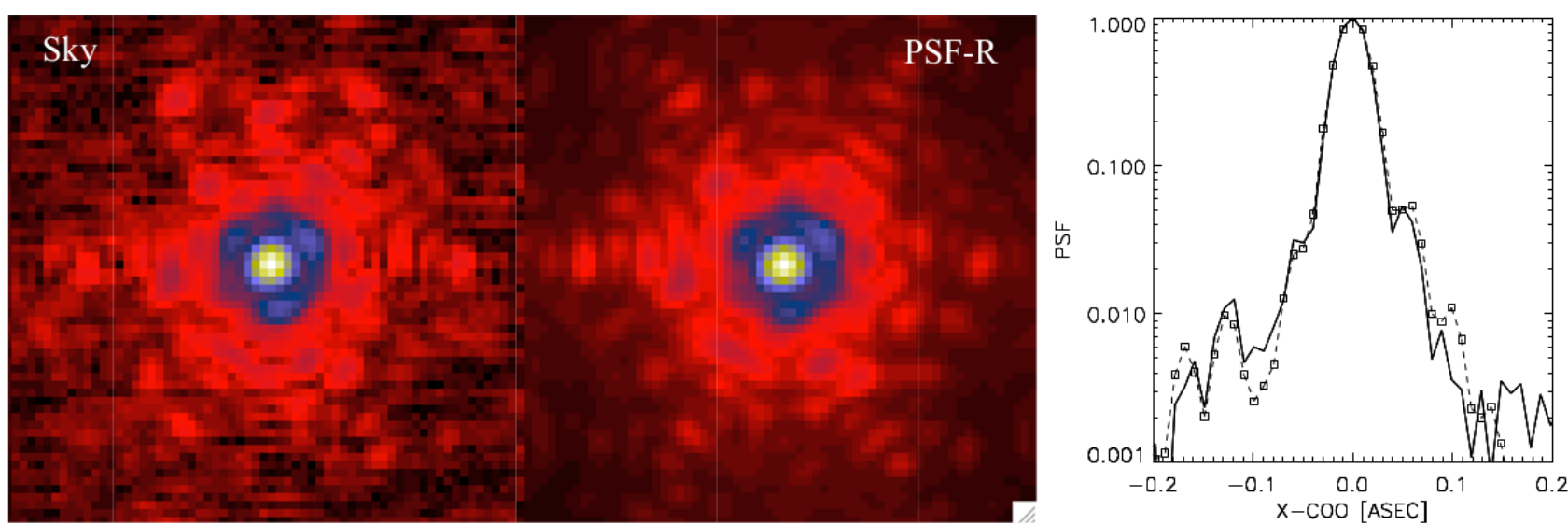

Figure 3: The reconstructed NGS PSF (middle) is compared with the target PSF (left) for a high Strehl ratio case $(50 \%)$. The images were taken at $1.655 \mu \mathrm{m}$. Right: a scan across the PSF through the peak is shown in logarithmic scale 25

${ }^{*}$ See L. Jolissaint presentation at AO4ASTRO: https://nuage.osupytheas.fr/s/9eS7WSXJ3ww57t5 


\section{- PSFAO19 model}

A recent analytical description has been proposed ${ }^{21,22}$ to represent the PSD/PSF from physical-based parameters. This model jointly describes both the corrected and uncorrected parts of the PSF, with successful recent applications. ${ }^{46}$ In comparison of the previous discussed techniques, this model simplifies the PSF determination problem thanks to his parsimony and can be used as a parametric model (focal-plane-based approach) or be calibrated from the AO control loop data (pupil-plane-based approach). The parsimony is a major advantage here: with only 7 parameters (no static aberrations accounted for), the model shown excellent match over on-sky-observations as presented in Figure 4 that presents a comparison of on-sky PSFs obtained on 7 different AO-assisted instruments with corresponding fitted models. The model adapts to any kind of $\mathrm{AO}$ correction; the differences are at the level of a few percents among all systems regardless the imaging wavelength (Vis./near infra-red) and the pixel scale (undersampling/oversampling). For over 4,800 PSFs obtained on these instruments, the model succeeds in reproducing the PSF metrics (Strehlratio, FWHM) at less than $4 \%$ or error. ${ }^{21}$ Note that this focal-plane-based/pupil-plane-based dual aspect could be also true for the Fourier-based model; we could distinguish a few parameters in this model to be retrieved on the image. Nevertheless, building such a model already requires AO expertise to describe the propagation of spatial frequencies, while the PSFAO19 model is a concise and parsimonious representation of the known AO PSD behavior, and still guarantee an excellent level of accuracy. The only drawback of the current implementation is that secondary parameters are not yet clearly connected to the AO control loop data, and seems to vary across the field and wavelength as well. This is an on-going analysis that is currently done in the framework of the ANR APPLY.



Figure 4: Two dimensional comparison in log scale and over $64 \times 64$ pixels of (top) observed PSFs, (middle) fitted model for multiple types of $\mathrm{AO}$ systems and instruments working in either visible or NIR wavelengths. From left to right: SPHERE/ZIMPOL, SPHERE/IRDIS, Keck AO/NIRC2, SOUL/LUCI, CANARY/CAMICAZ in MOAO mode, GALACSI/MUSE NFM, and GEMS/GSAOI. All PSFs have been normalized to the max.

\subsection{Hybrid reconstruction}

Hybrid PSF determination techniques gather both focal-plane, pupil-plane measurements and analytical models of atmospheric/instrumental defects for achieving the best reconstruction possible. The presence of a star in the field does not guarantee our capability to identify accurately the PSF shape at any position in the field, especially regarding field PSF variations, source crowding and noise contamination. Consequently, constraining the PSF shape from a model that inherits from the AO telemetry allows to calibrate the PSF reconstruction process. For instance, accounting for the spatial decorrelation of phase disturbances so-called anisoplanatism effect, ${ }^{47}$ and calibrating field-dependent static aberrations,${ }^{37}$ one may constrain the PSF shape at a point B in the field, knowing the PSF at a point A. Starting from this idea, a new Starfinder-based algorithm, so-called AIROPA, ${ }^{36,48,49}$ relies on a PSF extraction and characterization of PSF spatial variations through anisoplanatism models and calibration of field-dependent static aberrations. In a sense, it fits the hybrid reconstruction class regarding the level of expertise needed in $\mathrm{AO}$ models and calibration.

In the same spirit, the algorithm called PRIME ${ }^{27}$ combines PSF fitting and PSF reconstruction techniques to calibrate the PSF model. Contrary to PSF reconstruction, atmospheric and system parameters on which the 
model relies are no longer fixed; they can be retrieved on the focal-plane image directly, which guarantees an optimal use of the AO telemetry for fitting the PSF For instance, the seeing value is determined from the PSF wings. On data acquired with SPHERE/ZIMPOL in V and NIRC2 at Keck in H/K, a $1 \%$ of error on the 2D PSF estimation has been obtained. ${ }^{27,28}$ Hybrid techniques are particularly well suited to science cases that offer access to stars in the field.

\subsection{Marginal PSF estimation (\& deconvolution) from images of extended objects}

Deconvolution refers to technique that aim to restoring high-spatial frequencies that are filtered out through the optical system. ${ }^{50-53}$ In the isoplanetic domain, the imaging equation is

$$
\mathbf{i}=\mathbf{o} \star \mathbf{h}+\mathbf{n}
$$

where $i$ is the image, $o$ is the object, $h$ is the PSF and $n$ is an additive noise. Deconvolution is an estimation $\hat{o}$ of the object that produced the image $i$ and performed by the minimization of the following criterion

$$
\mathcal{J}(\mathbf{o}, \mathbf{h})=\|\mathbf{W}(\mathbf{i}-\mathbf{o} \star \mathbf{h})\|^{2}+\mu_{o} \mathcal{R}_{o}(\mathbf{o})+\mu_{h} \mathcal{R}_{h}(\mathbf{h})
$$

where the first term is the data-attached term that is the quadratic distance from the model to the data and weighted by the noise variance, ${ }^{50,52}$ and where $\mathcal{R}_{o}(\mathbf{o})$ and $\mathcal{R}_{h}(\mathbf{h})$ are penalty terms on respectively the object and the PSF. In practice, estimating jointly the object and the PSF (myopic deconvolution) is a highly undertermined problem and not feasible and the PSF can converge towards the Dirac function. ${ }^{54}$ Using a parametric model to describing the PSF and adjust jointly the object and PSF parameters also leads to algorithm degeneracy. ${ }^{50,51}$

The sucessful approaches so far consisted in providing a strong prior on the PSF, such as $\mathcal{R}_{h}(\mathbf{h})=0.5 \sum_{f} \mid \tilde{\mathbf{h}}-$ $\left.\tilde{\mathbf{h}}_{\mathbf{m}}\right|^{2} / S_{h}(f)$, with $\tilde{h}_{m}$ the mean OTF and $S_{h}(f)$ the associated spatial PSD, which must be estimated empirically.

To circumvent this need of particular PSF observations, a technique is currently being developed to estimate the PSF directly from the image of a resolved object. This technique is called "marginal PSF estimation" and has successfully been applied in the past for biomedical images. ${ }^{51}$ The approach has been adapted to astronomical images ${ }^{50}$ with direct applications to asteroid imaging for example. The basic principle of the method is to state that the observed image contains as much information on the object as on the PSF itself. Using a statistical approach, the object is integrated - marginalised - out of the problem to focus on the PSF estimation. A parametric model of the PSF is used so it reduces the number of unknowns to estimate by the marginal technique. Once the PSF parameters are known, we directly retrieve the PSF shape. Then this PSF is used to perform deconvolution of the image to restore the details of the observed object. In general, deconvolution processes are ill-posed problems highly sensitive to the PSF shape. The marginal PSF estimation provides an accurate PSF shape, usable for this difficult problem of deconvolution. The marginally estimated PSF may also be applied in any post-processing technique requiring the knowledge of the PSF.

\subsection{Discussion on uncertainties}

There is no real consensus on the estimation of uncertainties on the PSF through the PSF reconstruction process. Some work have been conducted in the past to address this question. ${ }^{55,56}$ In the case where the PSF is determined from an analytical model of the residual phase PSD (Fourier, covariance model-based), the PSF uncertainties are directly driven by the uncertainties on the model inputs parameters (seeing, $C_{n}^{2}(h), L_{0}, \ldots$ ) and one may easily establish this connection. Note that biases are (must be) compensated by comparing the PSF estimator with on-sky observations. ${ }^{23}$

Fig. 5 illustrates the photometric bias and precision obtained on simulated HARMONI PSF in SCAO mode, including the real ELT pupil and the cophasing error map. The PSF were obtain from the FAST simulator ${ }^{57}$ We have defined a distribution of seeing $\mathbf{S}=\mathcal{N}_{T}\left(\mu_{s}, \sigma_{s}\right)$, where $\mathcal{N}_{T}$ is a truncated normal distribution with $\mu_{s}=0.65^{\prime \prime}$ the mean-value of the distribution and the reference seeing value, $\sigma_{s}$ the precision we set, and $s>0 \quad \forall s \in \mathbf{S}$. We measured the distribution on the photometric error through a two-folds process: (i) compute $\mathrm{PSF}_{\sigma}$ from a selection $s \in \mathbf{S}$ with $\sigma_{s}$ defined from $1 \%$ to $50 \%$ of $\mu_{s}$, and (ii) perform a linear regression to obtain $\widehat{a}=\underset{a}{\operatorname{argmin}} \| \mathrm{PSF}_{0}-a \times\left.\mathrm{PSF}_{\sigma}\right|^{2}$, where $\mathrm{PSF}_{0}$ is the ground truth, and measure the photometric error as 

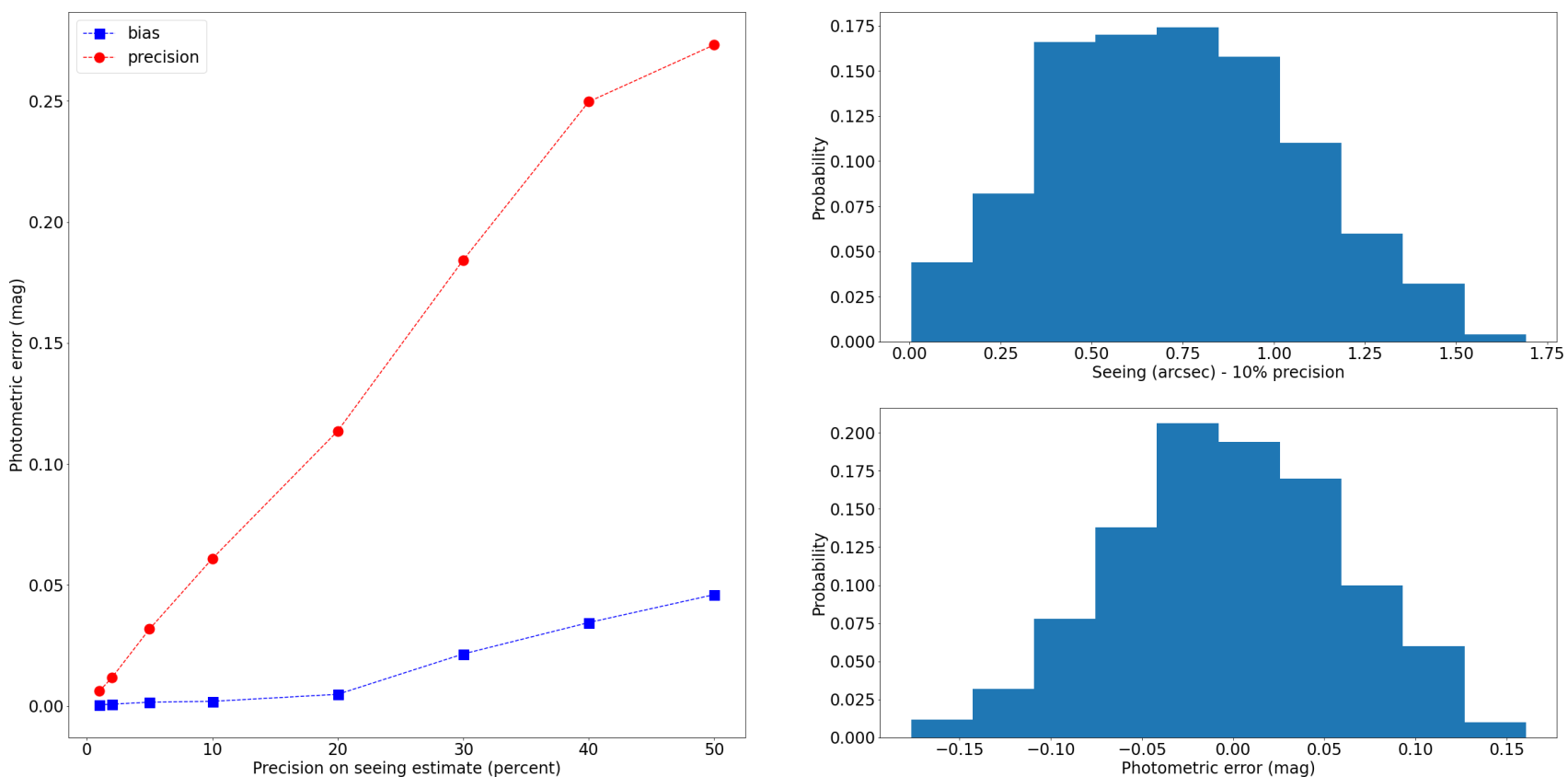

Figure 5: Left: H-band photometric bias and precision (1- $\sigma)$ obtained on simulated HARMONI PSFs with respect to the precision on the seeing estimates (bias on seeing estimate is assumed to be compensated). Right: histograms of the seeing and photometric error distributions for $\sigma_{s}=0.1 \mu_{s}$.

$\Delta m=-2.5 \log _{10}(\widehat{a})$. First of all, the distribution of the photometric error is $\mathcal{N}\left(\mu_{m}, \sigma_{m}\right)$, a Normal distribution as well, with a mean $\mu_{m}$ and standard-deviation $\sigma_{s}$ whose estimates are function of $\sigma_{s}$ as illustrated in Fig. 5 .

For the usual $10 \%$ precision we obtain on seeing measurements, ${ }^{58,59}$ we can obtain a marginally bias photometry measurements with a precision of $0.06 \mathrm{mag}$ at $1-\sigma$, accounting on the sole precision on the seeing estimation (no noise) and assuming that the PSF is perfectly described by the Fourier model. However, if WFS time series are used (PSF reconstruction), the seeing estimate impacts the PSF wings model only (we stick to the isoplanatic case), while in the present analysis, the seeing shapes the PSF cores as well. Considering that the Fourier model is a perfect representation of the PSF wings (actually the PSF wings are modeled using this approach regardless the pupil-plane determination technique), this methodology reveals the upper-bound on the photometric error due to seeing estimates precision.

If the reconstruction process is more complex, the estimation of the uncertainties is not completely straightforward, but still feasible if we identify what parameters are critical, such as the optical gains for instance or the seeing estimates. Other aspect, such as accuracy of the temporal transfer function model or even DM influence function shape, matter but might not be the main cause of the uncertainty, although this deserves a more complete analysis that will be conducted in the future.

\section{APPLICATIONS TO POST-PROCESSING}

\subsection{Characterization of brown dwarfs binary systems with PSF-R using NIRC2@Keck}

One of the two science cases identified for the first phase of science verification is low-mass binaries. The binaries used for our science verification tests are sourced from a sample that has been regularly monitored with Keck AO, both NGS and LGS, for essentially the entire history of these Keck facilities (e.g., $\left.;{ }^{60} ;{ }^{6162}\right)$. We used the most recent orbital analysis from ${ }^{63}$ to select binaries with precisely known orbits that can provide both on- and off-axis tests with NGS or LGS AO, although we have focused on testing on-axis PSF-R to date. Gl 569Bab (M8.5+M9) and HD 130948BC (L4+L4) can be observed as on-axis NGS targets because they are both in hierarchical triple systems with bright primary stars only 4.9" and 2.6" away, respectively. LP 349-25AB (M7+M8) and 2MASS J0746+2000AB (L0+L1.5) are an on-axis LGS targets. Of all these systems, Gl 569Bab is perhaps the most 
useful because its primary star is only 4 mag brighter than the binary components, so unsaturated images of all three components can be obtained, with the primary providing an empirical PSF reference only 4.9" off-axis.

Without PSF-R, fitting binary images requires either assuming an analytical PSF model (e.g., multi-component, elliptical Gaussians) or deriving an empirical PSF from the image itself (e.g., StarFinder ${ }^{1}{ }^{1}$. Generally, the former is necessary for the tightest binaries where the two components are barely distinguishable from a single elongated PSF, while for wider binaries with more cleanly separated component PSFs the StarFinder algorithm can successfully identify two stars and estimate the PSF that they share in common. The median astrometric error of tight (separations of 40 - 80 mas) binaries from ${ }^{63}$ that were predominantly fit using the analytic PSF model is 0.8 mas. In contrast, wider binaries with 100-200 mas separations analyzed with StarFinder yield typical errors of 0.1 mas ( $1 \%$ of a NIRC2 pixel). In terms of photometry, StarFinder yields flux ratios with a typical precision of $0.01 \mathrm{mag}$ as judged from the repeatability of the measurements across multiple epochs.

In principle, PSF-R should not significantly improve on StarFinder when the latter performs properly, regarding StarFinder capabilities to optimally determine the PSF without any theoretical assumptions about the atmosphere and telescope optics. Therefore, a reasonable goal of PSF-R would be to achieve 0.1 mas errors for the tightest binaries, i.e., accuracy comparable to what is possible when the PSF can be determined directly from the imaging data. The reconstructed PSFs are used in the science data analysis, in conjunction with the standard methods, to assess the performance. Essentially, binary-fitting analysis is performed for different cases such as using the reconstructed PSFs (PSF-R), the on-sky primary star PSF (GL569A), and the 2D Gaussian model. The resultant binary model parameters are compared with the reference values estimated from binary orbit fit in Tab. 1.

\begin{tabular}{|c|c|c|c|}
\hline & Binary separation (mas) & Position angle (degrees) & Delta magnitude (mag) \\
\hline Orbit & $67.18 \pm 0.24$ & $169.2 \pm 0.4$ & $0.47 \pm 0.04$ \\
\hline PSF-R & $66.7 \pm 0.8$ & $169.4 \pm 0.8$ & $0.46 \pm 0.03$ \\
\hline Gl 569 A & $66.9 \pm 0.6$ & $169.9 \pm 0.5$ & $0.53 \pm 0.03$ \\
\hline Gaussian & $67.1 \pm 1.0$ & $169.6 \pm 0.6$ & $0.64 \pm 0.05$ \\
\hline
\end{tabular}

Table 1: Binary parameters estimated from different methods using 40 exposures of NIRC2 in H-band.

These measurements, taken at just one binary separation, position angle and delta magnitude are not necessarily statistically representative of performance in general, but they do provide chance to look for possible issues. For example, the agreement between a simple Gaussian fit and the orbit astrometry is surprisingly good, but the disagreement in the magnitude difference shows that there is more to the story (e.g., perhaps the systematic errors in the relative astrometry cancel out in this fit better than those in the relative photometry). Such agreement between a simple 2D Gaussian model and true parameters are rarely seen in simulations of such binaries and thus require further investigations. The PSF-R results agree quite well with both the orbit baseline truth, and the fit using Gl 569A as the template. In particular, the magnitude difference is better reproduced by the PSF-R rather than the Gl 569A, indicating that the on-axis PSF-R may indeed be superior to the off-axis observed PSF as it includes the spatial variations of the PSF due to anisoplanatism and field-dependent static aberrations. ${ }^{37}$ Two other science cases, namely, the morphology of QSO host galaxies and microlensing, in addition to low-mass binaries, are considered for the next phase of science verification.

\subsection{Improving astrometry/photometry measurements on SPHERE/ZIMPOL}

As part of a technical proposal following the ESO Calibration Workshop $2017^{\dagger}$, Massari et. al observed the core of the Galactic globular cluster NGC 6121 with the AO assisted camera SPHERE/ZIMPOL ${ }^{64}$ in V-band, and under the program ID 60.A-9801(S). More details of the observation are given $\mathrm{in}^{65,66}$ The observed field illustrated in Fig. 6 contains only five stars in a 3.5 " $\times 3.5$ " field of view, where the brightest star has a Vband magnitude of $\mathrm{V}^{\prime}=10.9 \mathrm{mag}$, and has been used as the natural guide star for the AO system. Photometric measurements of the central star were obtained using four different techniques over 24 successive acquisitions, (i) aperture photometry, (ii) DAOPHOT-II, ${ }^{3}$ (iii) PSF-fitting using the PSFAO19 model, ${ }^{22}$ and (iv) PSF-fitting using PRIME. ${ }^{28}$

\footnotetext{
${ }^{\dagger}$ www.eso.org/sci/meetings/2017/calibration2017.html
} 



Figure 6: Left: Average of the SPHERE/ZIMPOL observations of NGC 6121. The five detected stars are marked with red circles. Right: Photometric (top panel) and astrometric (bottom panel) precision achieved on the guide star when using the aperture method (first column), DAOPHOT-II (second column), the PSFAO19 model (third column), and PRIME (last column).

The analysis illustrates that numerical PSF models, such as PRIME or PSFAO19, reach more precise measurements than the a-posteriori techniques, both photometrically (by a factor of 10) and astrometrically (by a factor of 4), advocating for the potential of innovative PSF-modeling methods as a powerful tool for analyzing future AO-assisted observations.

\subsection{Towards an upgrade of Starfinder illustrated on GeMS/GSAOI}

Another illustration of the potential of advanced PSF models for photometry/astrometry measurements using PSF-fitting is illustrated in Fig. 7, that shows astrometric error histograms and color-magnitude diagrams obtained by deploying Starfinder ${ }^{1,67,68}$ on R136 simulated images based on a GeMS/GSAOI configuration with a pixel scale of 20 mas/pixel in $\mathrm{H}$ and $\mathrm{K}$ bands. The PSF were simulated thanks to the Fourier PSF simulator ${ }^{16}$ which is broadly used in the community, in the HSIM simulator for HARMONI ${ }^{69}$ for instance.

Fig. 7 illustrates that the use of the numerical model PSFAO19, ${ }^{22}$ that is preliminary adjusted on simulated PSF, allows to unbias astrometry measurements and improve precision by a factor 4 , while photometric measurement accuracies are enhanced by up to a factor 10. A current work is focusing on an upgrade of Starfinder in order to include the analytical expression of the PSFAO19 model to improve the whole processing chain performed in Starfinder.

\subsection{Exploitation of MUSE NFM data with PampelMuse}

Advanced PSF models can also be employed in the analysis of integral field spectroscopy, for example obtained using MUSE $^{70}$ in the narrow field mode (NFM). MUSE NFM data of several globular clusters were obtained as part of the guaranteed time observations, in the star clusters program. ${ }^{71}$ In order to extract individual stellar spectra from the data, the reduced cubes are processed with the PAmpelMuse software. ${ }^{72}$ PampelMuse first tries to determine the PSF of the observation as well as the coordinates of the resolved sources as a function of wavelength. In a second step, this information is used to perform an optimal extraction of the spectrum of each resolved star. As illustrated in Fig. 2, the default PSF model used by PAMPELMUSE is a Moffat profile. While this profile provides a good representation of the actual PSF of MUSE data in the wide fied mode (WFM), it cannot account for the complexity of the NFM PSF. Therefore, the PSFAO19 ${ }^{22}$ model was implemented in PAMPElMuse. In Fig. 8, we illustrate the improvement in performance this model provides relative to a Moffat PSF in a MUSE NFM observation of the globular cluster NGC 5139. The central panel of Fig. 8 shows that even when the Moffat profile is complemented by a Gaussian core in order to represent the diffraction-limited part of the PSF, strong fit residuals around the bright stars remain. Instead, using the dedicated PSFAO19 model strongly suppresses these residuals. 

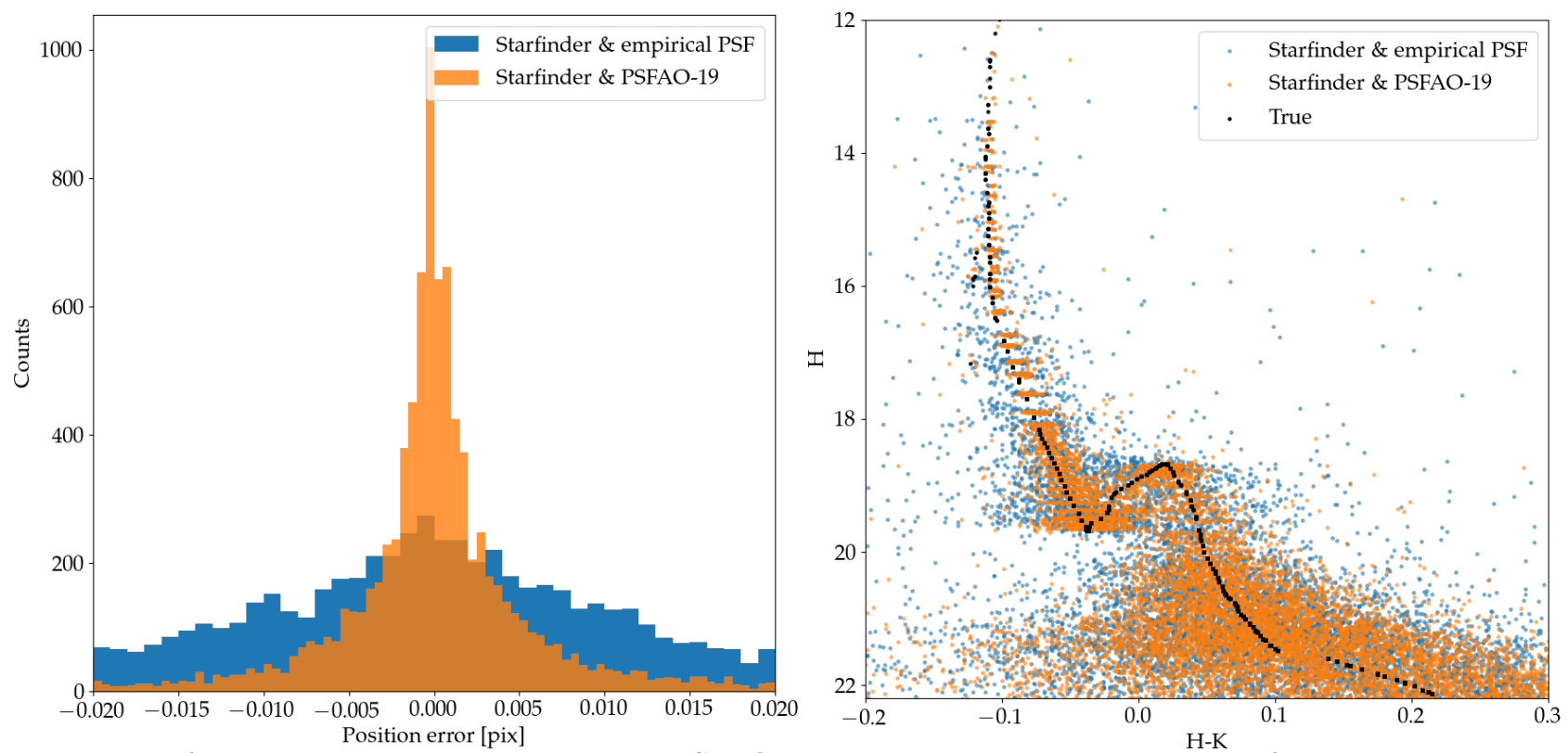

Figure 7: Left: astrometric error obtained with Starfinder applied to simulated images of R136 observed with GSAOI at Gemini, using the native PSF identification routine or the PSFAO19 model preliminary adjusted on simulated PSFs. Right: Corresponding color-magnitude diagrams. The empirical PSF is a median value of several PSF extracted from the image. In order to account for spatial variations, the image is segmented over $7 \times 7$ regions.

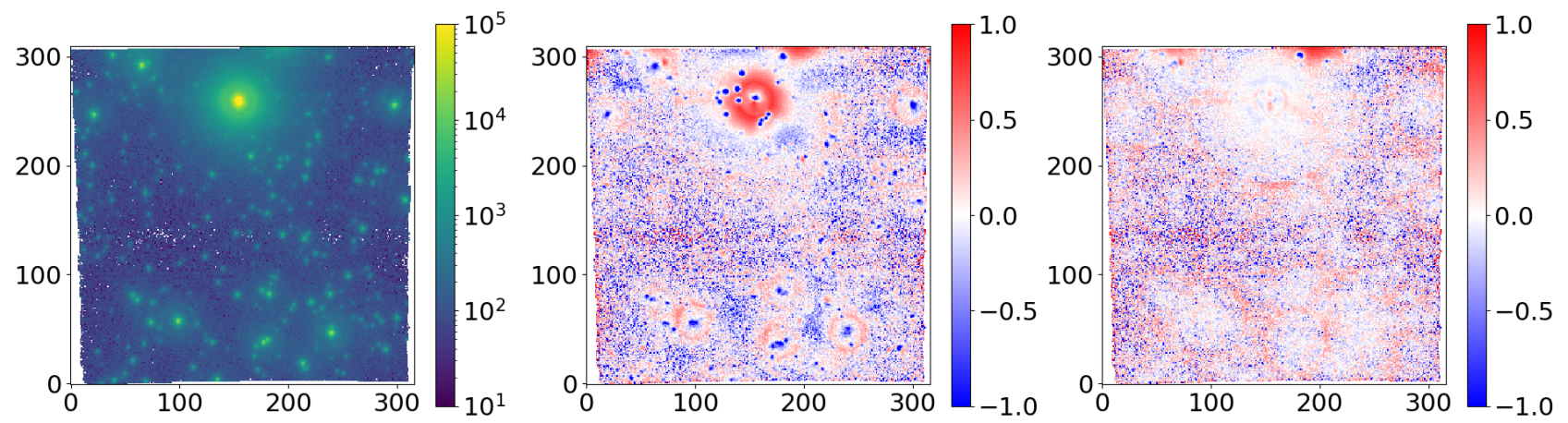

Figure 8: Left : Image of NGC5139 obtained with MUSE NFM at $850 \mathrm{~nm}$. Middle: residual map after stars detection and PSF subtraction using a Moffat-Gauss model for describing the PSF. Right: Residual map obtained using the PSFAO19 model. The mean residual error is improved by a factor 3 thanks to the new model definition.

\subsection{Deconvolution of asteroids images obtained with SPHERE/ZIMPOL}

The biggest asteroids of the Main Belt have been observed ${ }^{\ddagger}$ at high resolution with the VLT SPHERE/ZIMPOL imager. The SPHERE instrument benefits from the SAXO extreme adaptive optics system. Figure 9 (middle image) shows the observation ${ }^{46}$ of the asteroid Vesta with SPHERE/ZIMPOL on June 8th, 2018. This image represents the state-of-the-art ground based instruments with current extreme AO systems, on which the overall shape is captured but finest details are attenuated. The result of the deconvolution for Vesta can be seen on Fig. 9 (right). It exhibits sharp edges, and finer details such as local albedo variations or craters down to $20-30 \mathrm{~km}$ when observed from Earth at more than 170 million kilometers away.

\footnotetext{
${ }^{\ddagger}$ ESO Large Progam ID 199.C-0074 (PI: P. Vernazza)
} 


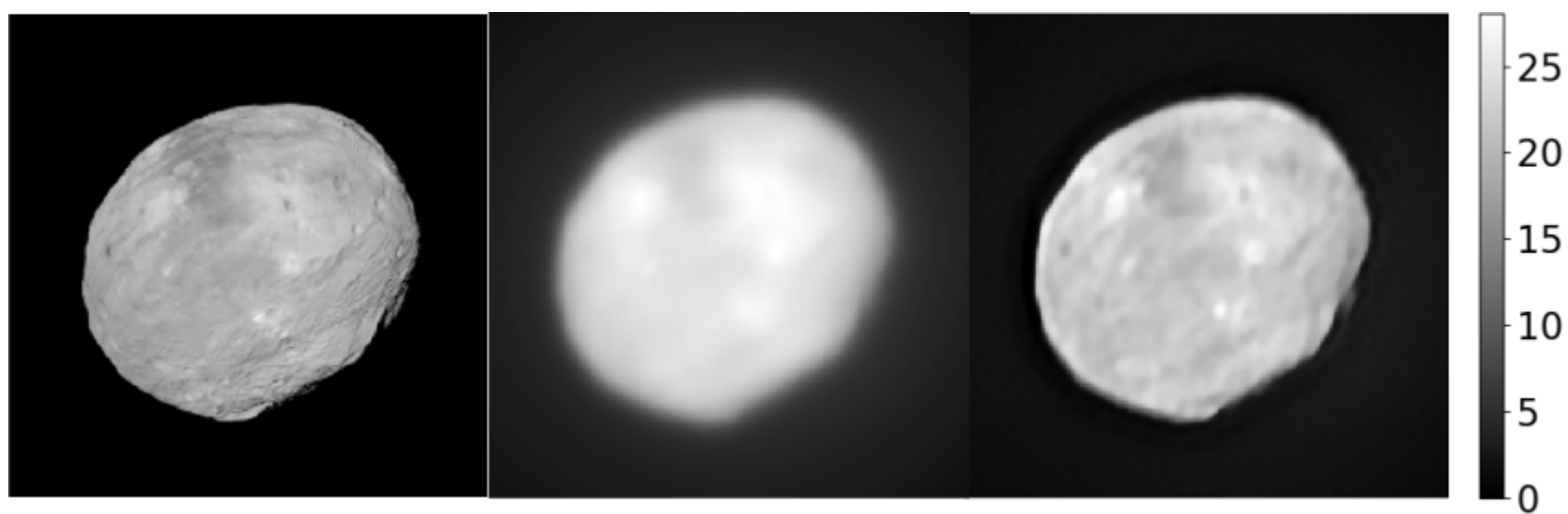

Figure 9: Left: Synthetic high-resolution image of VESTA produced by OASIS (L.Jorda, LAM) from the NASA/Dawn data. Middle : SPHERE/ZIMPOL observation in R band. Right : deconvolution using the marginal approach. ${ }^{50}$

\section{SUMMARY AND CONCLUSIONS}

We have classified the PSF determination techniques into three classes: (i) focal-plane-based techniques, (ii) pupil-plane-based techniques, and (iii) hybrid techniques that combine observed image and models of models of atmospheric/instrumental aberrations.

More particularly, PSF reconstruction refers to techniques that estimate or constrain the post-AO PSF from AO control loop data, e.g. time series of WFS/DM measurements and associated calibration. Multiple implementations (Fourier, covariance-based, reconstruction) may be envisioned, depending on the needs of PSF accuracy, RTC flexibility, data flow and data storage capabilities. On the one hand, there is the historical PSF reconstruction approach proposed by Véran et. al that derives the PSF, with a potential accuracy of $1 \%$ on PSF metrics or PSF mean square error, from the time-history of WFS measurements, which may be time and computational-power-demanding and constraining for the RTC data flow control. On the other hand, there are analytical and parsimonious techniques that require access to integrated parameters only $\left(\mathrm{seeing}, C_{n}^{2}(h)\right.$ profile, photons return,...) and allow to model the PSF with a few up to $10 \%$ of accuracy on PSF metrics.

From the past work that invoked PSF reconstruction for the processing of AO-corrected images, we distinguish several scenarios:

- Very accurate PSF determination for analysis of crowded regions. This seems to be the most demanding science case in terms of needed accuracy on the PSF. This is also a case for which stars are present in the field and the most efficient way to benefit both PSF reconstruction and phased-shifted (wavelength-shifted) representation of the PSF in the focal plane is to go for hybrid PSF reconstruction, which is expected to beat the $1 \%$ of accuracy on the PSF recovery in the field (and in wavelength as well).

- Moderate ( $10 \%$ ) up to very accurate PSF determination for the processing of images that contains stars. If point sources are observed in the field, an alternative way to calibrate the PSF model in the field is to rely on a parametric model of the PSF that also offers the possibility to take advantages of the spatial and spectral representation of the PSF. Such an approach is particularly meaningful when AO control loop data are not available, and also for tomographic AO systems for which an extension of the PSF reconstruction framework is still missing.

- Moderate PSF determination for the processing of stars-free astronomical images. In this situation, one must rely on AO control loop and use, either the classical PSF reconstruction recipe, or an analytical model. In both cases, the estimation process must be calibrated through a sample of on-sky observed PSF.

It is also crucial to keep in mind that PSF reconstruction can only reconstruct spatial frequencies to which the WFS is sensitive enough to deliver a linearly-dependent wavefront representation; non-linear wavefront sensors, 
high-order spatial frequencies and unseen frequencies (NCPA for instance) must be derived from alternative techniques. One of the advantage of parametric PSF models is that any fitting procedure is sensitive (noisedependent) to all spatial frequencies that feature the PSF shape and can be rendered thanks to an appropriate calibration. For instance, accurate photometry requires to model the AO PSF wings formed by uncompensated spatial frequencies; the use of the time-history WFS measurements is not needed in this situation as all pupilbased PSF determination techniques will rely on the same model; the PSF wings are derived from the Kolmogorov PSD model of the atmospheric phase that depends on the seeing estimation only, that maybe obtained from the AO control data or from the focal-plane image directly.

Finally, any decision to deploy such or another PSF reconstruction approach must absolutely be driven by science and user interface requirements before getting into technical implementation.

\section{ACKNOWLEDGMENTS}

This work has been partially funded by the French National Research Agency (ANR) program APPLY - ANR-19-CE31-0011. This work also benefited from the support of the WOLF project ANR-18-CE31-0018 of the and the OPTICON H2020 (2017-2020) Work Package 1.

\section{REFERENCES}

[1] Diolaiti, E., Bendinelli, O., Bonaccini, D., Close, L. M., Currie, D. G., and Parmeggiani, G., "StarFinder: A code for stellar field analysis." Astrophysics Source Code Library (Nov. 2000).

[2] Bertin, E. and Arnouts, S., "SExtractor: Software for source extraction.," A\&GA 117, 393-404 (June 1996).

[3] Stetson, P. B., "DAOPHOT - A computer program for crowded-field stellar photometry," PASP 99, 191-222 (Mar. 1987).

[4] Bernard, A., Neichel, B., Mugnier, L. M., and Fusco, T., "Optimal correction of distortion for high-angular-resolution images: Application to GeMS data," MNRAS 473, 2590-2607 (Jan. 2018).

[5] Massari, D., Fiorentino, G., McConnachie, A., Bono, G., Dall'Ora, M., Ferraro, I., Iannicola, G., Stetson, P. B., Turri, P., and Tolstoy, E., "GeMS MCAO observations of the Galactic globular cluster NGC 2808: the absolute age," A 8 A 586, A51 (Feb 2016).

[6] Yelda, S., Lu, J. R., Ghez, A. M., Clarkson, W., Anderson, J., Do, T., and Matthews, K., "Improving Galactic Center Astrometry by Reducing the Effects of Geometric Distortion," ApJ 725, 331-352 (Dec. 2010).

[7] Schödel, R., "Accurate photometry with adaptive optics in the presence of anisoplanatic effects with a sparsely sampled PSF. The Galactic center as an example of a challenging target for accurate AO photometry," A 8 A 509, A58 (Jan. 2010).

[8] Conan, R. and Correia, C., "Object-oriented Matlab adaptive optics toolbox," in [Adaptive Optics Systems IV], Proc. SPIE 9148, 91486C (Aug. 2014).

[9] Ferreira, F., Gendron, E., Rousset, G., and Gratadour, D., "Numerical estimation of wavefront error breakdown in adaptive optics," $A \& A$ 616, A102 (Aug. 2018).

[10] Basden, A. G., Bharmal, N. A., Jenkins, D., Morris, T. J., Osborn, J., Peng, J., and Staykov, L., "The Durham Adaptive Optics Simulation Platform (DASP): Current status," SoftwareX 7,63-69 (Jan 2018).

[11] Agapito, G., Puglisi, A., and Esposito, S., "PASSATA: object oriented numerical simulation software for adaptive optics," in [Proc. SPIE], Proc. SPIE 9909, 99097E (Jul 2016).

[12] Reeves, A., "Soapy: an adaptive optics simulation written purely in Python for rapid concept development," in $[P r o c$. SPIE], Proc. SPIE 9909, 99097F (Jul 2016).

[13] Rigaut, F. and Van Dam, M., "Simulating Astronomical Adaptive Optics Systems Using Yao," in [Proceedings of the Third AO4ELT Conference], Esposito, S. and Fini, L., eds., 18 (Dec 2013).

[14] Montilla, I., Béchet, C., Lelouarn, M., Correia, C., Tallon, M., Reyes, M., and Thiébaut, É., "Comparison of Reconstruction and Control algorithms on the ESO end-to-end simulator OCTOPUS," in [Adaptative Optics for Extremely Large Telescopes], 03002 (Jan 2010).

[15] Jolissaint, L., "Synthetic modeling of astronomical closed loop adaptive optics," Journal of the European Optical Society - Rapid publications, 5, $10055 \mathbf{5}$ (Nov. 2010).

[16] Neichel, B., Fusco, T., and Conan, J.-M., "Tomographic reconstruction for wide-field adaptive optics systems: Fourier domain analysis and fundamental limitations," Journal of the Optical Society of America A 26, 219 (Dec. 2008).

[17] Rigaut, F. J., Véran, J.-P., and Lai, O., "Analytical model for Shack-Hartmann-based adaptive optics systems," in [Adaptive Optical System Technologies], D. Bonaccini \& R. K. Tyson, ed., Proc. SPIE 3353, 1038-1048 (Sept. 1998).

[18] Beltramo-Martin, O., Correia, C. M., Mieda, E., Neichel, B., Fusco, T., Witzel, G., Lu, J. R., and Véran, J.-P., "Off-axis point spread function characterization in laser guide star adaptive optics systems," MNRAS 478, 4642-4656 (Aug. 2018).

[19] Britton, M. C., "The Anisoplanatic Point-Spread Function in Adaptive Optics," PASP 118, 885-900 (June 2006).

[20] Fried, D. L., "Limiting Resolution Looking Down Through the Atmosphere," Journal of the Optical Society of America (1917-1983) 56, 1380 (Oct. 1966).

[21] Beltramo-Martin, O., Fétick, R., Neichel, B., and Fusco, T., "Joint estimation of atmospheric and instrumental defects using a parsimonious point spread function model.On-sky validation using state of the art worldwide adaptive-optics assisted instruments," arXiv e-prints, arXiv:2009.00994 (Sept. 2020).

[22] Fétick, R. J. L., Fusco, T., Neichel, B., Mugnier, L. M., Beltramo-Martin, O., Bonnefois, A., Petit, C., Milli, J., Vernet, J., Oberti, S., and Bacon, R., "Physics-based model of the adaptive-optics-corrected point spread function. Applications to the SPHERE/ZIMPOL and MUSE instruments," A\&A 628, A99 (Aug 2019).

[23] Fusco, T., Bacon, R., Kamann, S., Conseil, S., Neichel, B., Correia, C., Beltramo-Martin, O., Vernet, J., Kolb, J., and Madec, P. Y., "Reconstruction of the ground-layer adaptive-optics point spread function for MUSE wide field mode observations," $A \xi A$ 635, A208 (Mar. 2020). 
[24] Ragland, S., Dupuy, T. J., Jolissaint, L., Wizinowich, P. L., Lu, J. R., van Dam, M. A., Berriman, G. B., Best, W., Gelino, C. R., Ghez, A. M., Liu, M. C., Mader, J. A., Vayner, A., Witzel, G., and Wright, S. A., "Status of point spread function determination for Keck adaptive optics," in [Adaptive Optics Systems VI], Proc. SPIE 10703, 107031J (July 2018).

[25] Ragland, S., Jolissaint, L., Wizinowich, P., van Dam, M. A., Mugnier, L., Bouxin, A., Chock, J., Kwok, S., Mader, J., Witzel, G., Do, T., Fitzgerald, M., Ghez, A., Lu, J., Martinez, G., Morris, M. R., and Sitarski, B., "Point spread function determination for Keck adaptive optics," in [Adaptive Optics Systems V], Proc. SPIE 9909, 99091P (July 2016).

[26] Jolissaint, L., Ragland, S., and Wizinowich, P., "Adaptive Optics Point Spread Function Reconstruction at W. M. Keck Observatory in Laser Natural Guide Star Modes : Final Developments," in [Adaptive Optics for Extremely Large Telescopes IV (AO4ELT4)], E93 (Oct. 2015).

[27] Beltramo-Martin, O., Correia, C. M., Ragland, S., Jolissaint, L., Neichel, B., Fusco, T., and Wizinowich, P. L., "PRIME: PSF Reconstruction and Identification for Multiple-source characterization Enhancement - application to Keck NIRC2 imager," MNRAS 487, 5450-5462 (Aug 2019).

[28] Beltramo-Martin, O., Fusco, T., Neichel, B., Marasco, A., Massari, D., and J., M., "Pushing Point spread function reconstruction to the next level. Application to SPHERE/ZIMPOL.," MNRAS , 1256 (Dec 2019).

[29] Roddier, F., "The effects of atmospheric turbulence in optical astronomy," Progress in optics. Volume 19. Amsterdam, North-Holland Publishing Co., 1981, p. 281-376. 19, 281-376 (1981).

[30] Jolissaint, L., Mugnier, L. M., Neyman, C., Christou, J., and Wizinowich, P., "Retrieving the telescope and instrument static wavefront aberration with a phase diversity procedure using on-sky adaptive optics corrected images," in [Adaptive Optics Systems III], Ellerbroek, B. L., Marchetti, E., and Véran, J.-P., eds., Society of Photo-Optical Instrumentation Engineers (SPIE) Conference Series 8447, 844716 (July 2012).

[31] Mugnier, L. M., Sauvage, J.-F., Fusco, T., Cornia, A., and Dandy, S., "On-Line Long-Exposure Phase Diversity: a Powerful Tool for Sensing Quasi-Static Aberrations of Extreme Adaptive Optics Imaging Systems," Optics Express 16, 18406 (Oct. 2008).

[32] Blanc, A., Fusco, T., Hartung, M., Mugnier, L. M., and Rousset, G., "Calibration of NAOS and CONICA static aberrations. Application of the phase diversity technique," $A \mathscr{E} A$ 399, 373-383 (Feb. 2003).

[33] Lamb, M. P., Correia, C., Sauvage, J.-F., Véran, J.-P., Andersen, D. R., Vigan, A., Wizinowich, P. L., van Dam, M. A., Mugnier, L., and Bond, C., "Quantifying telescope phase discontinuities external to adaptive optics systems by use of phase diversity and focal plane sharpening," Journal of Astronomical Telescopes, Instruments, and Systems 3, 039001 (July 2017).

[34] Andersen, T., Owner-Petersen, M., and Enmark, A., "Image-based wavefront sensing for astronomy using neural networks," Journal of Astronomical Telescopes, Instruments, and Systems 6, 034002 (July 2020).

[35] Paine, S. W. and Fienup, J. R., "Machine learning for avoiding stagnation in image-based wavefront sensing," in [Image Sensing Technologies: Materials, Devices, Systems, and Applications VI], Proc. SPIE 10980, 109800T (May 2019).

[36] Ciurlo, A., Do, T., Witzel, G., Lu, J., Turri, P., Fitzgerald, M., Lyke, J., Correia, C., and Guerra, J. C., "PSF reconstruction for integral field spectrographs," Proc. SPIE (2020).

[37] Sitarski, B. N., Witzel, G., Fitzgerald, M., Meyer, L., Ghez, A. M., Campbell, R. D., Lu, J. R., Matthews, K., Wizinowich, P., and Lyke, J., "Modeling instrumental field-dependent aberrations in the nirc2 instrument on the keck ii telescope," in [Adaptive Optics Systems IV], Proc. SPIE 9148 (August 2014).

[38] Correia, C. M. and Teixeira, J., "Anti-aliasing Wiener filtering for wave-front reconstruction in the spatial-frequency domain for highorder astronomical adaptive-optics systems," Journal of the Optical Society of America A 31, 2763 (Dec. 2014).

[39] Bond, C. Z., Correia, C. M., Sauvage, J.-F., El Hadi, K., Neichel, B., and Fusco, T., "Fourier wavefront reconstruction with a pyramid wavefront sensor," in [Proc. SPIE], Proc. SPIE 10703, 107034M (Jul 2018).

[40] Martin, O. A., Correia, C. M., Gendron, E., Rousset, G., Gratadour, D., Vidal, F., Morris, T. J., Basden, A. G., Myers, R. M., and Neichel, B.and Fusco, T., "PSF reconstruction validated using on-sky CANARY data in MOAO mode," in [Adaptive Optics V for proc. SPIE], (2016).

[41] Gendron, É., Charara, A., Abdelfattah, A., Gratadour, D., Keyes, D., Ltaief, H., Morel, C., Vidal, F., Sevin, A., and Rousset, G., [A novel fast and accurate pseudo-analytical simulation approach for MOAO], vol. 9148 of Proc. SPIE, 6 (Aug. 2014 ).

[42] Villecroze, R., Fusco, T., Bacon, R., and Madec, P.-Y., "PSF reconstruction for MUSE in wide field mode," in [Adaptive Optics Systems $I I I]$, Proc. SPIE 8447, 84475W (July 2012).

[43] Veran, J.-P., Rigaut, F., Maitre, H., and Rouan, D., "Estimation of the adaptive optics long-exposure point-spread function using control loop data.," Journal of the Optical Society of America A 14, 3057-3069 (Nov. 1997).

[44] Clénet, Y., Lidman, C., Gendron, E., Rousset, G., Fusco, T., Kornweibel, N., Kasper, M., and Ageorges, N., "Tests of the PSF reconstruction algorithm for NACO/VLT," in [Proc. SPIE], Proc. SPIE 7015, 701529 (Jul 2008).

[45] Gilles, L., Correia, C., Véran, J.-P., Wang, L., and Ellerbroek, B., "Simulation model based approach for long exposure atmospheric point spread function reconstruction for laser guide star multiconjugate adaptive optics," Appl. Opt. 51, 7443-7458 (Nov 2012).

[46] Fétick, R. J., Jorda, L., Vernazza, P., Marsset, M., Drouard, A., Fusco, T., Carry, B., Marchis, F., Hanuš, J., Viikinkoski, M., Birlan, M., Bartczak, P., Berthier, J., Castillo-Rogez, J., Cipriani, F., Colas, F., Dudziński, G., Dumas, C., Ferrais, M., Jehin, E., Kaasalainen, M., Kryszczynska, A., Lamy, P., Le Coroller, H., Marciniak, A., Michalowski, T., Michel, P., Mugnier, L. M., Neichel, B., Pajuelo, M., Podlewska-Gaca, E., Santana-Ros, T., Tanga, P., Vachier, F., Vigan, A., Witasse, O., and Yang, B., "Closing the gap between Earth-based and interplanetary mission observations: Vesta seen by VLT/SPHERE," A\&A 623, A6 (Mar 2019).

[47] Fried, D. L., "Anisoplanatism in adaptive optics," Journal of the Optical Society of America (1917-1983) 72, 52 (Jan. 1982).

[48] Turri, P., Lu, J., Ciurlo, A., Do, T., Ghez, A., Witzel, G., and Britton, M., "Testing PSF reconstruction on sky with AIROPA," Proc. SPIE (2020).

[49] Witzel, G., Lu, J. R., Ghez, A. M., Martinez, G. D., Fitzgerald, M. P., Britton, M., Sitarski, B. N., Do, T., Campbell, R. D., Service, M., Matthews, K., Morris, M. R., Becklin, E. E., Wizinowich, P. L., Ragland, S., Doppmann, G., Neyman, C., Lyke, J., Kassis, M., Rizzi, L., Lilley, S., and Rampy, R., "The AIROPA software package: milestones for testing general relativity in the strong gravity regime with AO," in [Adaptive Optics Systems V], Proc. SPIE 9909, 990910 (July 2016).

[50] Fétick, R. J. L., Mugnier, L. M., Fusco, T., and Neichel, B., "Blind deconvolution in astronomy with adaptive optics: the parametric marginal approach," MNRAS 496, 4209-4220 (July 2020).

[51] Blanco, L. and Mugnier, L. M., "Marginal blind deconvolution of adaptive optics retinal images," Optics Express 19, 23227 (Nov. 2011).

[52] Mugnier, L. M., Fusco, T., and Conan, J.-M., "MISTRAL: a myopic edge-preserving image restoration method, with application to astronomical adaptive-optics-corrected long-exposure images," Journal of the Optical Society of America A 21, 1841-1854 (Oct. 2004). 
[53] Mugnier, L. M., Robert, C., Conan, J.-M., Michau, V., and Salem, S., "Myopic deconvolution from wave-front sensing," Journal of the Optical Society of America A 18, 862-872 (Apr 2001).

[54] Thiébaut, E. and Conan, J.-M., "Strict a priori constraints for maximum-likelihood blind deconvolution," J. Opt. Soc. Am. A 12, 485-492 (Mar 1995).

[55] Jolissaint, L., Carfantan, H., and Anterrieu, E., "Exploring the impact of PSF reconstruction errors on the reduction of astronomical adaptive optics based data," in [Adaptive Optics Systems], Proc. SPIE 7015, 70152K (July 2008).

[56] Exposito, J., Gratadour, D., Clénet, Y., Rousset, G., and Mugnier, L., [Estimation of errors on the PSF reconstruction process for myopic deconvolution], vol. 8447 of Proc. SPIE (July 2012).

[57] Neichel, B., Beltramo-Martin, O., Plantet, C., Rossi, F., Guido, A., Carolo, E., Fusco, T., Cirasuolo, M., and van der Burg, R., "A NEW TOOL TO EFFICIENTLY PREDICT YOUR FAVORITE AO PSF," Proc. SPIE (2020).

[58] Andrade, P. P., Garcia, P. J. V., Correia, C. M., Kolb, J., and Carvalho, M. I., "Estimation of atmospheric turbulence parameters from Shack-Hartmann wavefront sensor measurements," MNRAS 483, 1192-1201 (Feb 2019).

[59] Jolissaint, L., Ragland, S., Christou, J., and Wizinowich, P., "Determination of the optical turbulence parameters from the adaptive optics telemetry: critical analysis and on-sky validation," Appl. Opt. 57, 7837 (Sept. 2018).

[60] Lane, B. F., Zapatero Osorio, M. R., Britton, M. C., Martín, E. L., and Kulkarni, S. R., "The Orbit of the Brown Dwarf Binary Gliese 569B," ApJ 560, 390-399 (Oct. 2001).

[61] Dupuy, T. J., Liu, M. C., Bowler, B. P., Cushing, M. C., Helling, C., Witte, S., and Hauschildt, P., "Studying the Physical Diversity of Late-M Dwarfs with Dynamical Masses," ApJ 721, 1725-1747 (Oct. 2010).

[62] Konopacky, Q. M., Ghez, A. M., Barman, T. S., Rice, E. L., Bailey, J. I., I., White, R. J., McLean, I. S., and Duchêne, G., "Highprecision Dynamical Masses of Very Low Mass Binaries," ApJ 711, 1087-1122 (Mar. 2010).

[63] Dupuy, T. J. and Liu, M. C., "Individual Dynamical Masses of Ultracool Dwarfs," ApJS 231, 15 (Aug. 2017 ).

[64] Schmid, H. M., Bazzon, A., Roelfsema, R., Mouillet, D., Milli, J., Menard, F., Gisler, D., Hunziker, S., Pragt, J., and Dominik, C., "SPHERE/ZIMPOL high resolution polarimetric imager. I. System overview, PSF parameters, coronagraphy, and polarimetry," $A \& A$ 619, A9 (Nov 2018).

[65] Massari, D., Beltramo-Martin, O., Marasco, A., Milli, J., Fiorentino, G., Neichel, B., Fusco, T., Tolstoy, E., and Kerber, F., "Precise photometry and astrometry of the globular cluster NGC 6121 using PSF-R techniques," Proc. SPIE (2020).

[66] Massari, D., Marasco, A., Beltramo-Martin, O., Milli, J., Fiorentino, G., Tolstoy, E., and Kerber, F., "Successful application of PSF-R techniques to the case of the globular cluster NGC 6121 (M 4)," arXiv , arXiv:2001.08134 (2020).

[67] Schreiber, L., Diolaiti, E., and Beltramo-Martin, O., "Starfinder2: a software package for identification and analysis of point-like sources in Adaptive Optics images with spatially variable PSF," Proc. SPIE (2020).

[68] Schreiber, L., Diolaiti, E., Sollima, A., Arcidiacono, C., Bellazzini, M., Ciliegi, P., Falomo, R., Foppiani, I., Greggio, L., Lanzoni, B., Lombini, M., Montegriffo, P., Dalessandro, E., and Massari, D., "Developing a new software package for PSF estimation and fitting of adaptive optics images," in [Adaptive Optics Systems III], Proc. SPIE 8447, 84475V (July 2012).

[69] Zieleniewski, S., Thatte, N., Kendrew, S., Houghton, R. C. W., Swinbank, A. M., Tecza, M., Clarke, F., and Fusco, T., "HSIM: a simulation pipeline for the HARMONI integral field spectrograph on the European ELT," MNRAS 453, 3754-3765 (Nov. 2015).

[70] Bacon, R., Accardo, M., Adjali, L., Anwand, H., Bauer, S., Biswas, I., Blaizot, J., Boudon, D., Brau-Nogue, S., and Brinchmann, J., "The MUSE second-generation VLT instrument," Proc. SPIE 7735, 773508 (Jul 2010).

[71] Kamann, S., "PampelMuse: Crowded-field 3D spectroscopy," (May 2018).

[72] Kamann, S., Wisotzki, L., and Roth, M. M., "Resolving stellar populations with crowded field 3D spectroscopy," A\& A 549, A71 (Jan. 2013). 